\title{
Insuficiência cardíaca grave de etiologia amiloidótica
}

\author{
Severe heart failure of amylootic etiology
}

\section{Gustavo Henrique Belarmino de Góes ${ }^{1}$, Mirella Valença Mota Vianna ${ }^{1}$, Diana Patricia Lamprea Sepulveda ${ }^{2}$, Dário Celestino Sobral Filho ${ }^{3}$}

Góes GHB, Vianna MVM, Sepulveda DPL, Sobral Filho DC. Insuficiência cardíaca grave de etiologia amiloidótica / Severe heart failure of amylootic etiology. Rev Med (São Paulo). 2018 maio-jun.;97(3):368-71.

RESUMO: A amiloidose é uma doença sistêmica causada pela deposição extracelular de fibrilas insolúveis de proteínas de baixo peso molecular em diversos tecidos. Pode ser classificada nas formas: primária, secundária, hereditária e sistêmica senil. Relatamos o caso de um paciente masculino, 75 anos, que deu entrada em emergência cardiológica com quadro progressivo de edema em membros inferiores iniciado há quatro meses. Trinta dias antes do internamento passou a apresentar dispneia de médios para pequenos esforços, evoluindo para dispneia ao repouso e em decúbito (insuficiência cardíaca - classe funcional IV). Ao exame físico apresentou-se em estado geral regular, consciente, taquidispneico, afebril, hidratado, hipocorado $(1+/ 4+)$, ictérico $(2+/ 4+)$, acianótico e presença de estase jugular a $45^{\circ}$. O ritmo cardíaco era regular em dois tempos e bulhas, normofonéticas, com sopro sistólico em foco mitral $(2+/ 6+)$ desdobramento fixo da segunda bulha; pressão arterial de $80 \times 40 \mathrm{mmHg}$ e frequência cardíaca de 84 bpm. O eletrocardiograma de admissão mostrou ritmo sinusal regular, bloqueio atrioventricular de $1^{\circ}$ grau, baixa voltagem em derivações de plano frontal, zona eletricamente inativa em região ântero-septal e distúrbio de condução pelo ramo esquerdo do feixe de His. Radiografia de tórax com área cardíaca aumentada, padrão de congestão pulmonar e derrame pleural bilateral, mais acentuado à esquerda. Também foi realizado ecocardiograma transtorácico que evidenciou câmaras cardíacas aumentadas à direita, função sistólica de ventrículo direito levemente deprimida, insuficiência tricúspide moderada a importante, hipertensão pulmonar moderada, hipertrofia de ventrículo esquerdo, disfunção de ventrículo esquerdo e achado de ecogenicidade granular brilhante. Ultrassonografia de abdome mostrou fígado e baço com textura heterogênea, sugestivo de doença granulomatosa. Posteriormente foi realizada biópsia hepática que confirmou a hipótese de doença infiltrativa determinada pela amiloidose cardiovascular. Paciente evoluiu com rebaixamento de nível de consciência e insuficiência renal aguda, sendo realizada hemodiálise e transferência para Unidade Coronariana, onde apresentou parada cardiorrespiratória e evolução para óbito.

Descritores:Insuficiência cardíaca/etiologia; Amiloidose/ etiologia; Idoso.

ABSTRACT: Amyloidosis is a systemic disease caused by the extracellular deposition of insoluble fibrils of low molecular weight proteins in various tissues. It can be classified in the forms: primary, secondary, hereditary and senile systemic. We report the case of a 75-year-old male patient who was admitted to a cardiologic emergency with progressive edema in the lower limbs started four months ago. Thirty days before admission

1. Universidade de Pernambuco (UPE), Faculdade de Ciências Médicas, Recife, PE, BR. Estudante de Medicina. Email: gustavogoesmt@ hotmail.com, mirella.valenca@gmail.com. ORCID: Góes GHB - https://orcid.org/0000-0003-3366-1182; Vianna MVM - https:// orcid.org/0000-0002-4355-5650.

2. Pronto-Socorro Cardiológico de Pernambuco (PROCAPE/ UPE), Recife, PR, BR. Chefia Médica da Enfermaria de Doenças Valvares, Preceptora e Vice-Coordenadora da Residência de Cardiologia. ORCID: Sepulveda DPL - https://orcid.org/0000-0001-8055-2128 Email: lampreadiana@gmail.com.

3. Universidade de Pernambuco (UPE), Recife, PE, BR. Professor Associado e Livre-Docente de Cardiologia; Coordenador Médico do Hospital Universitário PROCAPE - UPE. Fellow do American College of Cardiology e da European Society of Cardiology. Email: dsobral@uol.com.br. ORCID: Sobral Filho DC - https://orcid.org/0000-0002-5301-7741.

Autor correspondente: Gustavo Góes. Rua Arnóbio Marques, 310. Santo Amaro. Recife, PE, BR. CEP: 50100-130. Email: gustavogoesmt@gmail.com. 
he presented dyspnea from medium to small effort, evolving to restlessness and in decubitus (cardiac failure - functional class IV). On physical examination he presented a regular, conscious, tachidispneic, no fever, hydrated, hypochromic $(1+/ 4+)$, icteric $(2+/ 4+)$, acyanotic and jugular stasis at $45^{\circ}$. The cardiac rhythm was regular in two times and normal heart sounds, with systolic murmur in the mitral focus $(2+/ 6+)$ fixed unfolding of the second sound; blood pressure of $80 \times 40 \mathrm{mmHg}$ and heart rate of $84 \mathrm{bpm}$. The admission electrocardiogram showed regular sinus rhythm, 1st degree atrioventricular block, low voltage in frontal plane leads, electrically inactive zone in anteroseptal region and left bundle branch conduction disorder. Chest X-ray with increased cardiac area, pattern of pulmonary congestion and bilateral pleural effusion, more pronounced on the left. A

\section{INTRODUÇÃO}

Amiloidose é uma doença sistêmica causada pela deposição extracelular, na forma amiloide, de fibrilas insolúveis de proteínas de baixo peso molecular em diversos tecidos. Pode ser classificada nas formas: primária, secundária, hereditária e sistêmica senil. Idade acima de 50 anos, sexo masculino, antecedentes de doenças crônicas infecciosas ou inflamatórias e história familiar são os principais fatores predisponentes para essa condição ${ }^{1,2}$.

A forma primária é a amiloidose sistêmica mais comum, com média de idade de diagnóstico aos 60 anos e predominância masculina de 2:1. O acometimento cardíaco está presente em cerca de $50 \%$ dos pacientes, cursando com rápida evolução dos sintomas e mau prognóstico. Uma vez realizado o diagnóstico, a sobrevida média é de seis meses ${ }^{3}$.

\section{RELATO DE CASO}

Paciente masculino, 75 anos, deu entrada em emergência cardiológica com quadro progressivo de edema em membros inferiores iniciado há quatro meses, e progressão da dispneia para ortopneia nos últimos trinta dias (insuficiência cardíaca - classe funcional IV). Paciente hipertenso e com epidemiologia positiva para Doença de Chagas e Esquistossomose, fazendo uso regular de hidroclorotiazida $25 \mathrm{mg}$ / dia há dois anos, quando foi diagnosticado com hipertensão arterial sistêmica. Ao exame físico apresentou-se em estado geral regular, consciente, taquidispneico, afebril, hidratado, hipocorado $(1+/ 4+)$, ictérico $(2+/ 4+)$, acianótico e presença de estase jugular a $45^{\circ}$. Os murmúrios vesiculares estavam presentes em ambos hemitórax, porém diminuídos em bases e com creptos finos até o terço médio à direita; frequência respiratória de 24 ipm e saturação arterial de oxigênio de $92 \%$. Na avaliação cardiovascular constatou-se ictus cordis visível e palpável no $5^{\circ}$ espaço intercostal esquerdo, ocupando uma polpa digital; sem impulsão de meso. O ritmo cardíaco era regular em dois tempos e bulhas, normofonéticas, com sopro sistólico em foco mitral $(2+/ 6+)$ e desdobramento fixo da segunda bulha; pressão arterial de 80x40 mmHg e transthoracic echocardiogram was performed too, showing right enlarged cardiac chambers, mildly depressed right ventricular systolic function, moderate to severe tricuspid insufficiency, moderate pulmonary hypertension, left ventricular hypertrophy, left ventricular dysfunction and bright granular echogenicity finding. Ultrasonography of abdomen showed liver and spleen with heterogeneous texture, suggestive of granulomatous disease. Subsequently, hepatic biopsy was performed, confirming the hypothesis of infiltrative disease determined by cardiovascular amyloidosis. Patient evolved with lowered level of consciousness, and acute renal failure, undergoing hemodialysis and transfer to Coronary Unit, where he presented cardiorespiratory arrest and evolution to death.

Keywords: Heart failure/etiology; Amyloidosis/etiology; Aged.

frequência cardíaca de 84 bpm. O abdome estava globoso, flácido e doloroso abaixo do rebordo costal direito e apêndice xifoide. Fígado palpável a $5 \mathrm{~cm}$ abaixo do rebordo costal direito, presença de edema de parede abdominal e sinais sugestivos da presença de liquido ascítico. Ao exame das extremidades apresentou pulsos presentes e simétricos nos quatro membros, edema de membros inferiores (MMII) $(2+/ 4+)$ sem empastamento de panturrilhas. Para avaliação complementar foram realizados exames laboratoriais (Tabela 1), eletrocardiograma (ECG) (Figura 1) (ritmo sinusal regular, bloqueio atrioventricular de $1^{\circ}$ grau, baixa voltagem em derivações de plano frontal, zona eletricamente inativa em região anterosseptal e distúrbio de condução pelo ramo esquerdo do feixe de His), radiografia de tórax (Figura 2) (área cardíaca aumentada, padrão de congestão pulmonar e derrame pleural bilateral, mais acentuado à esquerda). $\mathrm{O}$ ecocardiograma transtorácico (ETT) mostrou câmaras cardíacas aumentadas à direita, função sistólica de ventrículo direito (VD) levemente deprimida, insuficiência tricúspide importante, hipertensão pulmonar moderada, disfunção de VE (fração de ejeção de 44\%), dilatação biatrial e achado de ecogenicidade granular brilhante. Continuando a investigação, foi realizada ultrassonografia de abdome que evidenciou fígado e baço com ecotextura heterogênea e aspecto sugestivo de doença granulomatosa, além de derrame pleural à esquerda e ascite leve. Na tomografia de tórax destaca-se o tamanho normal do coração e a preservação das estruturas vasculares, componentes de consolidação e atelectasia no lobo inferior esquerdo, foco com atenuação em vidro fosco no segmento lingular inferior, com padrão inflamatório-infeccioso e derrame pleural de moderado volume bilateral. Radiografia de abdome mostrou distensão de alças abdominais e ausência de gás e ampola retal. Com a suspeita de doença de depósito foi solicitada biópsia hepática, que confirmou tratar-se de amiloidose. Foi iniciada, então, terapia diurética com espironolactona, porém dois dias após o paciente evoluiu com potássio sérico de $5,58 \mathrm{mEq} / \mathrm{L}$; foi optado, então, por suspensão da espironolactona e iniciada furosemida EV para diminuição do edema de MMII, além de simeticona e antiemético, evoluindo na enfermaria 
com dificuldade para compensação clínica. Mesmo com a substituição do diurético, apresentou insuficiência renal aguda, com necessidade de realização de hemodiálise. Paciente evoluiu com rebaixamento de nível de consciência, sendo transferido para Unidade Coronariana, onde broncoaspirou e apresentou parada cardiorrespiratória com evolução para óbito.

Tabela 1 - Exames laboratoriais

\begin{tabular}{lcc}
\hline Variável & $\begin{array}{c}\text { Valor } \\
\text { encontrado }\end{array}$ & $\begin{array}{c}\text { Valor de } \\
\text { referência }\end{array}$ \\
\hline Hematócrito & $27 \%$ & $36-50 \%$ \\
\hline Hemoglobina $(\mathrm{g} / \mathrm{dL})$ & 9,9 & $12-17$ \\
\hline Leucócitos $\left(\mathrm{n}^{\circ} / \mathrm{mm}^{3}\right)$ & $11,3 \times 10^{3}$ & $5-11 \times 10^{3}$ \\
\hline Plaquetas $\left(\mathrm{n}^{\circ} / \mathrm{mm}^{3}\right)$ & $148 \times 10^{3}$ & $150-400 \times 10^{3}$ \\
\hline INR & 1,32 & $\leq 1,0$ \\
\hline Ureia $(\mathrm{mg} / \mathrm{dL})$ & 93 & $15-45$ \\
\hline Creatinina $(\mathrm{mg} / \mathrm{dL})$ & 3,21 & $0,5-1,3$ \\
\hline Sódio $(\mathrm{mEq} / \mathrm{L})$ & 128 & $135-145$ \\
\hline Potássio $(\mathrm{mEq} / \mathrm{L})$ & 4,48 & $3,5-5,5$ \\
\hline Cloreto $(\mathrm{mEq} / \mathrm{L})$ & 95,2 & $95-105$ \\
\hline PCR $(\mathrm{mg} / \mathrm{dL})$ & 142 & $<0,5$ \\
\hline Cálcio $(\mathrm{mg} / \mathrm{dL})$ & 9,25 & $8,5-10,5$ \\
\hline Albumina $(\mathrm{g} / \mathrm{dL})$ & 2,65 & $3,5-5,0$ \\
\hline Sumário de Urina & Ausência de & Ausência de \\
nitrito & $3+/ 4$ & Austrito \\
\hline Proteinúria & $4+/ 4$ & Ausente \\
\hline
\end{tabular}

Legenda: INR: Razão normalizada internacional; PCR: proteína C reativa.

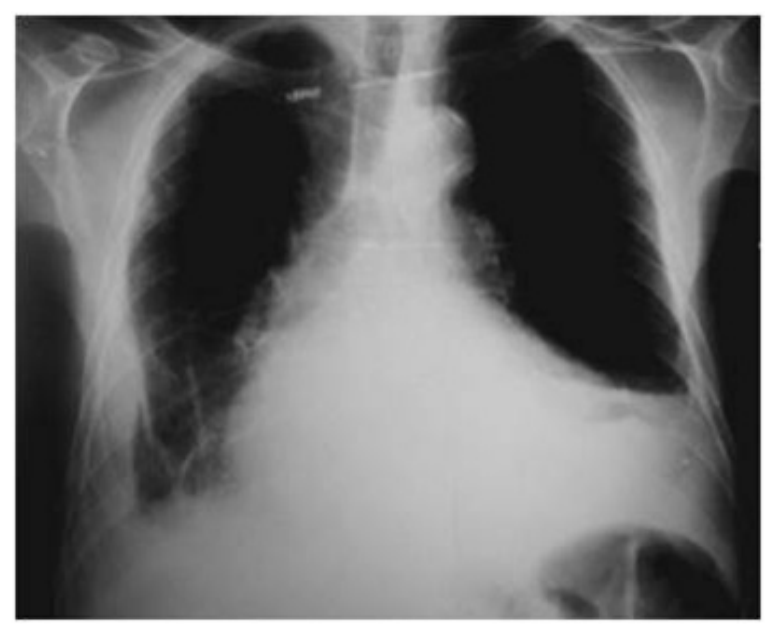

Figura 2 - Radiografia de tórax evidenciando área cardíaca aumentada, padrão de congestão pulmonar e derrame pleural bilateral, mais acentuado à esquerda

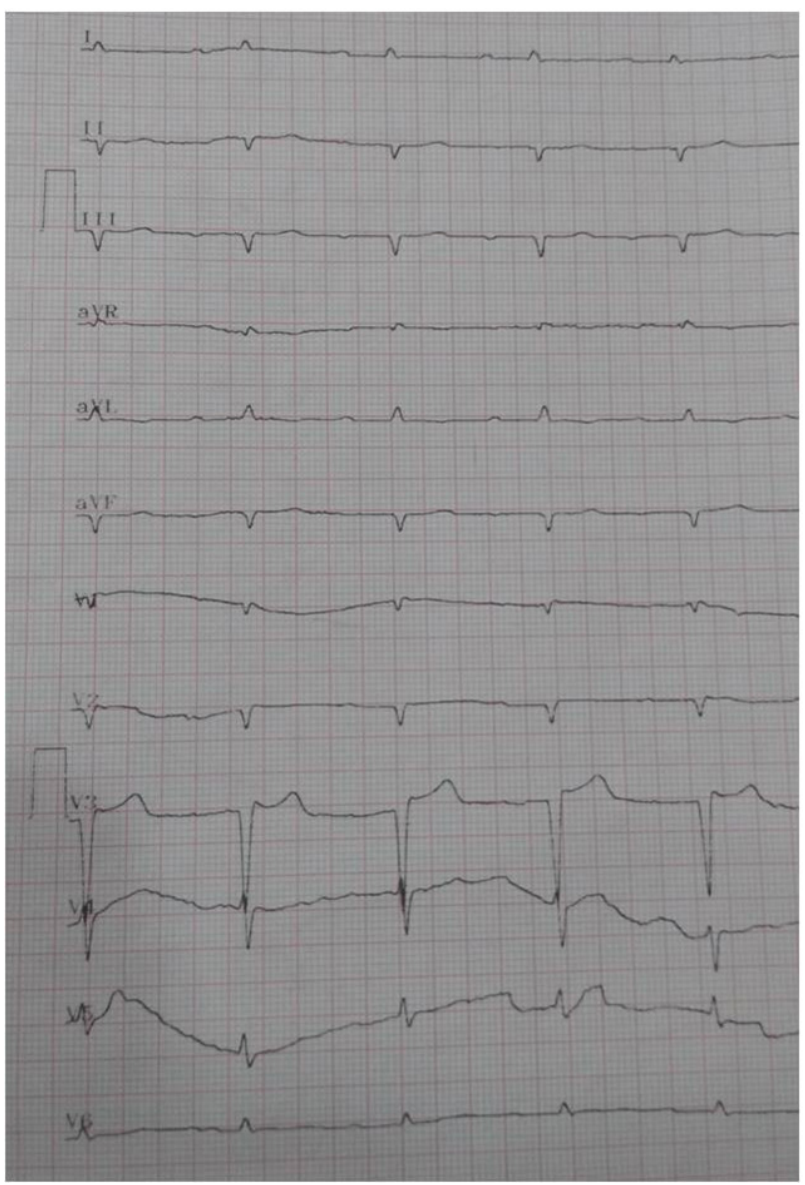

Figura 1 - Eletrocardiograma mostrando ritmo sinusal regular, bloqueio atrioventricular de $1^{\circ}$ grau, baixa voltagem em derivações de plano frontal, zona eletricamente inativa em região anterosseptal e distúrbio de condução pelo ramo esquerdo do feixe de His

\section{DISCUSSÃO}

Descrita pela primeira vez por Rudolph Virchow, em $1954^{1}$, a amiloidose é causada pela deposição extracelular de fibrilas insolúveis de proteínas de baixa densidade, sendo classificada como sistêmica quando há deposição dessas proteínas em vários órgãos. Já a amiloidose localizada ocorre quando a deposição restringe-se a um único órgão. Outra forma de classificação divide a amiloidose em: 1) primária (podendo ocorrer isoladamente); 2) secundária ou adquirida (o depósito ocorre como complicação de infecções ou inflamações crônicas); 3) hereditária ${ }^{1}$. Assim, a amiloidose cardíaca surge da deposição da proteína amiloide no tecido cardíaco, sendo a miocardiopatia restritiva a sua apresentação mais comum².

As manifestações clínicas dependem dos órgãos afetados, embora sintomas inespecíficos, como fadiga e/ ou anorexia, possam ocorrer. A amiloidose sistêmica geralmente apresenta como primeiro sinal a proteinúria, devido ao acometimento renal. Órgãos como fígado, rins e coração podem sofrer aumento de tamanho tanto na 
amiloidose primária quanto na secundária² .

Não é possível estabelecer um padrão de apresentação clínica da amiloidose cardiovascular, devendo-se suspeitar da doença quando insuficiência cardíaca crônica incontrolável se desenvolve em pacientes com mais de 50 anos, principalmente se associada a sinais de miocardiopatia restritiva, como distúrbios de condução, baixo débito cardíaco e comprometimentos atriais isolados. Além disso, deve-se buscar comprometimento de outros órgãos, pois a forma localizada da amiloidose só ocorre em cerca de 4\% dos $\operatorname{casos}^{3}$. Os achados clínicos de edema ascendente e progressivo associado a hepatomegalia, como no presente caso, sugerem acometimento predominantemente do coração direito, chamando a atenção para o diagnóstico de cardiopatia com restrição diastólica ${ }^{4}$.

O padrão-ouro para o diagnóstico de amiloidose cardíaca é a biópsia endomiocárdica. Embora associada a baixo risco de complicações (menor que 1\%), pode ocorrer: perfuração cardíaca com tamponamento, complicação vascular significativa, necessidade de cirurgia cardíaca de emergência e óbito. Por isso na prática clínica a biópsia endomiocárdica é pouco utilizada ${ }^{3}$. Rahman et al. ${ }^{5}$ compararam a eficácia diagnóstica do ECG e do ecocardiograma, em conjunto, à biópsia endomiocárdica em pacientes com amiloidose cardíaca, constatando que de fato a biópsia apresenta melhores resultados diagnósticos.

Apesar desses resultados, o ECG é importante para estabelecer o diagnóstico de amiloidose. O achado mais comum ao ECG é a baixa voltagem no plano frontal e baixa voltagem do complexo QRS em derivações dos membros ${ }^{6}$, além de distúrbios de condução ${ }^{7}$, como observado no presente caso. Outra alteração frequente é o padrão de pseudoinfarto. Menos frequentemente pode ocorrer fibrilação atrial paroxística ou persistente e áreas eletricamente inativas?.

Outro importante método diagnóstico é a ecocardiografia, que pode mostrar aumento de espessura das paredes ventriculares direita e/ ou esquerda. A visualização de aumento de espessura da parede do ventrículo esquerdo ao ecocardiograma associada ao padrão eletrocardiográfico de baixa voltagem apresenta alta especificidade para amiloidose cardíaca. Mesmo em pacientes assintomáticos, a ecocardiografia se mostrou sensível para detectar acometimento cardíaco na amiloidose ${ }^{8}$. Embora pouco frequente, o achado de ecogenicidade granular brilhante apresenta alta especificidade ${ }^{9}$, como ocorreu no presente caso.

A ressonância nuclear magnética cardíaca também é considerada um bom método diagnóstico, podendo identificar o espessamento miocárdico e do septo interatrial, sinais de disfunção diastólica e um padrão de realce tardio subendocárdico no $\mathrm{VE}^{3}$. No caso relatado, a ressonância chegou a ser solicitada, mas o paciente evoluiu para óbito antes da realização do exame.

O tratamento da insuficiência cardíaca é realizado basicamente pela administração de diuréticos. Os glicosídeos cardíacos geralmente não representam benefício para o quadro clínico do paciente, pois na maioria dos casos de amiloidose predomina a disfunção diastólica. Bloqueadores do canal de cálcio, inibidores da enzima conversora de angiotensina, bem como os betabloqueadores frequentemente induzem a hipotensão, limitando seu uso para esses pacientes. Quanto à abordagem cirúrgica (realização de transplante cardíaco) para o tratamento da amiloidose, não há consenso na literatura, não estando indicado em casos avançados nem nos casos de amiloidose sistêmica ${ }^{10}$.

Contribuição dos autores: Design do estudo: Góes GHB, Sobral Filho DC; Aquisição de dados: Góes GHB, Vianna MVM, Sepulveda DPL; Análise dos dados: Góes GHB, Vianna MVM, Sepulveda DPL, Sobral Filho DC; Desenvolvimento e edição do manuscrito: Góes GHB, Vianna MVM, Sepulveda DPL, Sobral Filho DC.

\section{REFERÊNCIAS}

1. Monteiro NF, Diz MCE. Dificuldades no diagnóstico da amiloidose primária: relato de caso. Rev Med Minas Gerais. 2015;25 (2):280-6. doi: 10.5935/2238-3182.20150048.

2. Fernandes A, Caetano F, Almeida I, Paiva L, Gomes P, Mota P, et. al. Amiloidose cardíaca - abordagem diagnóstica, a propósito de um caso clínico. Rev Port Cardiol. 2016;35(5):305. https://doi.org/10.1016/j.repc.2016.01.004.

3. Oberger JV, Filho CND, Garcia LA, Cordeiro MU. Amiloidose cardíaca isolada: relato de caso. Rev Bras Cardiol. 2014;27(3):213-16. http://dx.doi.org/10.11606/issn.21767262.v50i2p123-129.

4. Patel KS, Hawkins PN. Cardiac amyloidosis: where are we today? J Intern Med. 2015;278(2):126-44. doi: 10.1111/ joim. 12383.

5. Rahman JE, Helou EF, Gelzer-Bell R, Thompson RE, Kuo C, Rodriguez ER, et al. Noninvasive diagnosis of biopsy-proven cardiac amyloidosis. J Am Coll Cardiol. 2004;43:410-5. doi: 10.1016/j.jacc.2003.08.043.

6. Maleszewski JJ. Cardiac amyloidosis: pathology, nomenclature, and typing. Cardiovasc Pathol. 2015;24(6):34350. doi: 10.1016/j.carpath.2015.07.008.

7. Zhao L, Fang Q. Recent advances in the noninvasive strategies of cardiac amyloidosis. Heart Fail Rev. 2016;21(6):703-21. doi: 10.1007/s10741-016-9580-5.

8. Koyama J, Ikeda SI, Ikeda U. Echocardiographic assessment of the cardiac amyloidoses. Circulation. 2015;79(4):721-34. doi: 10.1253/circj.CJ-14-1425.

9. Noordzij W, Glaudemans AW, Longhi S, Slart RH, Lorenzini M, Hazenberg B P, Rapezzi C. Nuclear imaging for cardiac amyloidosis. Heart Fail Rev. 2015;20(2):145-54. doi: 10.1007/s10741-014-9463-6.

10. White JA, Fine NM. Recent advances in cardiovascular imaging relevant to the management of patients with suspected cardiac amyloidosis. Cur Cardiol Rep. 2016;18(8):77. doi: 10.1007/s11886-016-0752-7.

Submetido em: 18.02 .18

Aceito em: 12.04.18 